\title{
Vertebrobasilar artery insufficiency in rheumatoid atlantoaxial subluxation
}

\author{
M. W. JONES AND J. C. E. KAUFMANN \\ From the Department of Pathology (Neuropathology), University Hospital, \\ London, Ontario, Canada
}

SYNOPSIS Cervical myelopathy has become commonly recognized as a complication of rheumatoid atlantoaxial subluxation. A small group of patients with atlantoaxial subluxation may have intermittent symptoms associated with change of head position and which are due to vertebral artery compression. Two such cases are reported, one with necropsy findings of infarction in the area supplied by the vertebrobasilar system. The pathogenesis of the symptomatology and infarction is discussed.

There are two important neurological syndromes that can arise from rheumatoid atlantoaxial subluxation (AAS). One is a compression myelopathy (Stevens et al., 1971; Smith et al., 1972) which can uncommonly be complicated by acute compression with tetraplegia or death (Davis and Markley, 1951). The other less common syndrome is that of ischaemic symptoms related to vertebrobasilar artery insufficiency. We have recently encountered two cases of this latter syndrome, one of which came to postmortem examination. Pathological reports are rare but a comparable case was reported by Webb et al. (1968). This was a 53 year old female with AAS and vertebral artery thrombosis whose symptoms were intermittent.

\section{CASE 1}

Mrs. M.H. is a 45 year old lady with a 15 year history of seropositive rheumatoid arthritis. Six months before admission she had one week of confused amnestic behaviour of uncertain aetiology. Since then, she has had repeated bouts of vertigo, as interpreted by a neurologist, without other symptoms associated with neck flexion or head rotation. On the day of admission she fell unconscious and had bilateral clonic movements. In the emergency room

Address for correspondence: Dr J. C. E. Kaufmann, Department of Pathology (Neuropathology), Health Sciences Centre, University of Western Ontario, London, Ontario, Canada N6A 3K7.

(Accepted 18 September 1975.) the aetiology of her semicomatose state was not apparent. Forced flexion of her neck and back in preparation for a lumbar puncture provoked
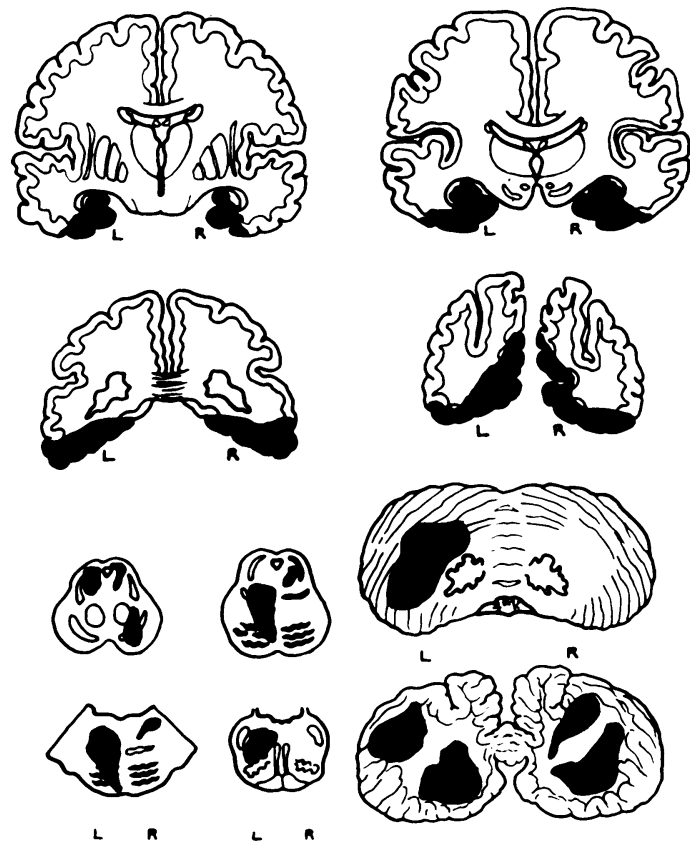

FIG. 1. Case 2. Diagram of infarcted areas in the distribution of the posterior cerebral artery territories as well as scattered infarcts in cerebellum and brainstem. Some infarcts were older than others. 


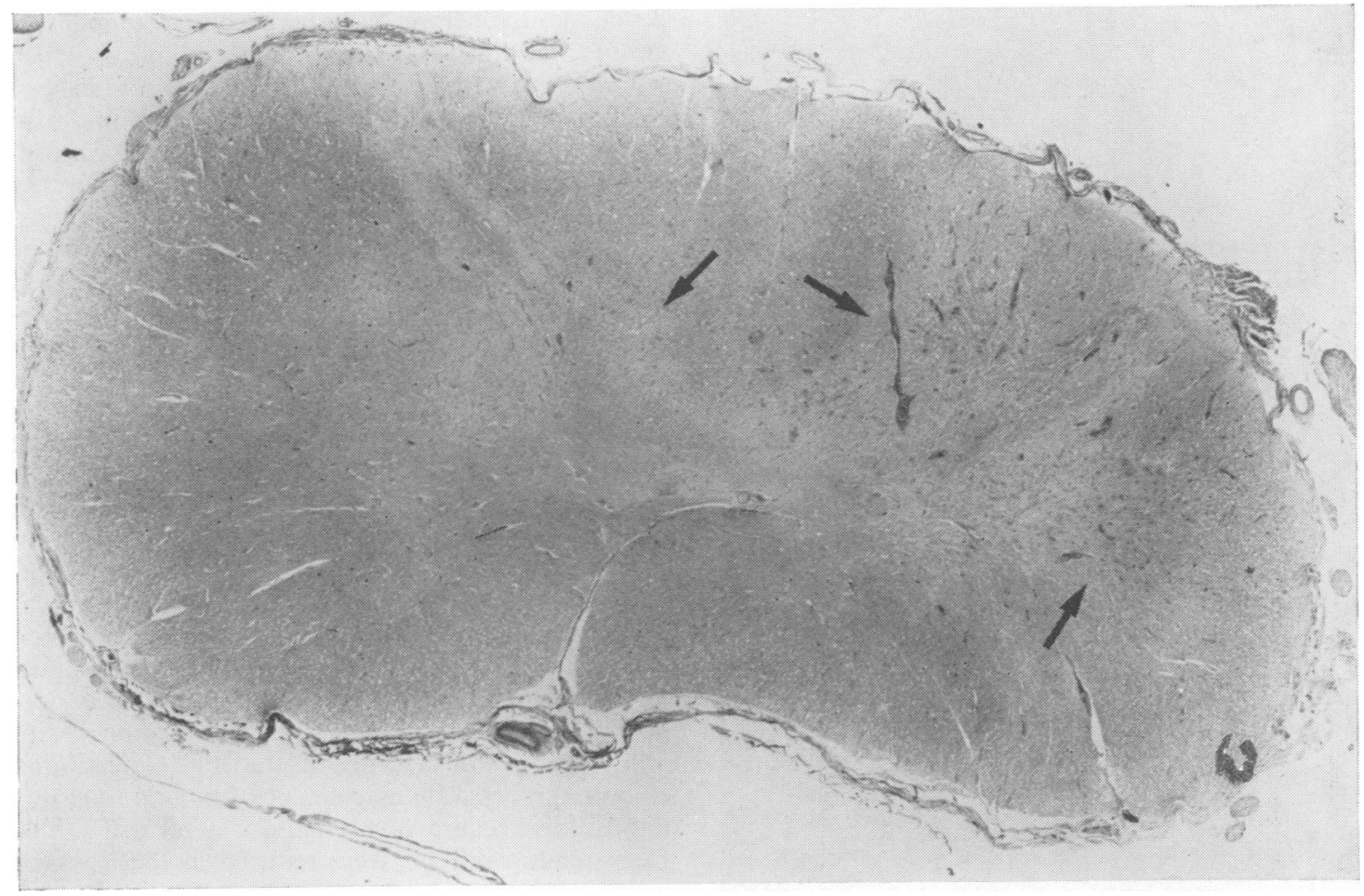

FIG. 2. Case 2. High cervical cord showing areas of infarction affecting grey and white matter (arrows). Luxol-fast blue and cresyl-violet, about $\times 2.5$, enlarged photographically.

opisthotonos. Bilateral carotid angiograms were normal; however, on retrospect AAS was seen. The following morning the patient was alert but confused and bilateral vertebral angiograms revealed normal vessels with no signs of impaired flow at the C1-C2 intervertebral space. Ten millimetres of displacement between the odontoid process and the anterior arch of $\mathrm{C} 1$ vertebra was noted. The patient inadvertently flexed her neck in the recovery room and again exhibited opisthotonos and lapsed into a stuporous state from which she awoke through phases of confusion and hallucination. At all examinations, her brain-stem reflexes, cranial nerves, deep tendon reflexes, muscle tone, and plantar reflexes were considered normal. A posterior fusion was performed wiring $\mathrm{C} 1$ to $\mathrm{C} 2$ vertebra (Galli technique). It was not possible to get close opposition and there was a $9.5 \mathrm{~mm}$ gap from the anterior arch of $\mathrm{Cl}$ vertebra to the odontoid process. There was an $11 \mathrm{~mm}$ canal behind the odontoid process.

The patient was seen 19 months postoperatively and had had no more symptoms of vertigo on neck flexion or rotation.
SUMMARY Her symptoms of vertigo seemed solely related to head flexion or rotation. We are suggesting that her comatose state and the mental confusion that followed were secondary to vertebral artery narrowing from the AAS. The vertebral angiogram was done when she was alert and her neck no longer moved enough to impair blood flow. In retrospect, the week of confusion six months before admission probably reflected a small stroke in her posterior cerebral artery territory (Mathew and Meyer, 1974). Tight opposition of $\mathrm{Cl}$ vertebra to the odontoid process was impossible at surgery. Chun et al. (1974) reported on the presence of a thick pannus of rheumatoid tissue present between the arch of $\mathrm{C} 1$ vertebra and the odontoid process acting as a mass lesion preventing close opposition. In their case, it was necessary to remove the abnormal tissue through an oral approach but in our case this was not necessary as her symptoms were primarily vascular and not those of a compressive myelopathy.

\section{CASE 2}

Mr. W.D.M. was a 74 year old man with a 30 year 


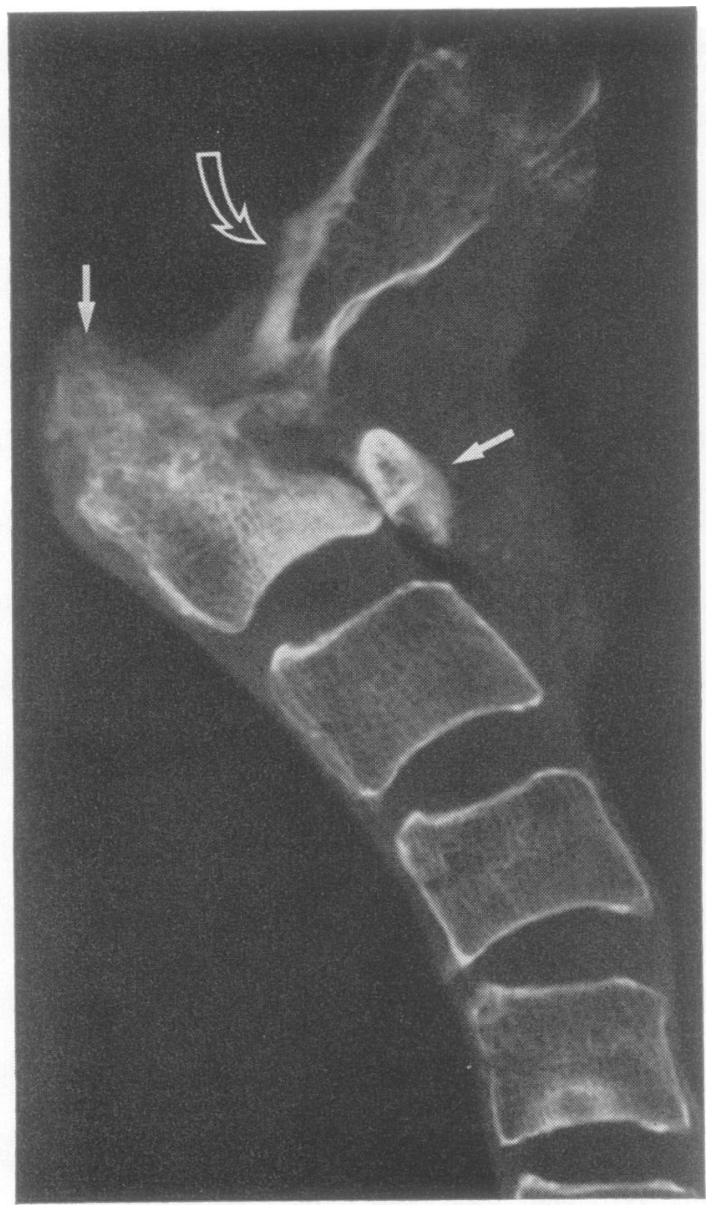

FIG. 3. Case 2. This lateral radiograph of the dissected necropsy specimen shows upward herniation of the odontoid process (arrow) through the foramen magnum above the basi-occiput (curved arrow) better than films made during life. The posterior part of the spinal column and foramen magnum have been removed. Note arch of atlas (arrow). history of typical deforming rheumatoid arthritis. Seven months before death he began to complain of vertigo, as interpreted by a neurologist, which would occur with flexion and/or rotation of his head to the right. In addition to vertigo, he would also describe a presyncopal feeling on arising in the morning which would be relieved by lying down. During this time, there were several falls with unconsciousness lasting for minutes but these could not be characterized further and were not investigated. In the month preceding admission he developed progressive weakness and paraesthesia of all four limbs and by the time of admission was unable to walk because of weakness and could no longer do up his buttons. He also complained bitterly about a constant suboccipital head pain.

Examination revealed a wasted emaciated man who had an orthostatic drop in blood pressure from $190 / 110$ to $110 / 80 \mathrm{mmHg}$. with symptoms of lightheadedness. He was certain that this sensation was different from his vertiginous feeling. He had been on antihypertensive medicine, which was discontinued on admission. Cranial nerve examination was normal. There was a moderate global weakness with mild flexion contractures of the hips, knees, and elbows. An accurate assessment of muscle tone was impossible because of his painful deformed joints. Deep tendon reflexes were reduced in the legs and normal in the arms; plantar reflexes were equivocal.

Radiography showed anterior subluxation of $\mathrm{Cl}$ and $C 2$ vertebrae and an upward herniation of the odontoid process through the foramen magnum, giving a picture of pseudobasilar invagination. On the 11th hospital day, while upright, the patient suddenly became unconscious for five minutes. He awoke and remained restless, confused, and disorientated. He now had right lateral gaze nystagmus, bilateral extensor plantar reflexes, and inability to hold his legs up against gravity. He was put in traction with skull tongs and his mental state and weakness were definitely improving when on the 29th hospital day he suddenly became unconscious and was deeply comatose with decerebrate posturing and Cheyne-Stokes respirations. His level of consciousness improved over the following days and he

FIG. 4. Case 2. View of the interior of posterior fossa with dorsum sellae above and confluence of the sinuses below. Note the odontoid process of the axis well into the posterior fossa (black arrow) and displaced to the right side. It lies immediately in front of the lower brain-stem and upper spinal cord which are still in situ. (white arrow).

FIG. 5. Case 2. Dissected specimen of foramen magnum area viewed from behind. The pedicles and laminae of the vertebrae have been removed. Note the herniated odontoid process of the axis projecting into the posterior fossa and the constriction of the right vertebral artery (arrow). An arrow indicates the cut surface of the rim of the foramen magnum on each side. The structure above the label is the dural sheath enclosing the spinal cord. 

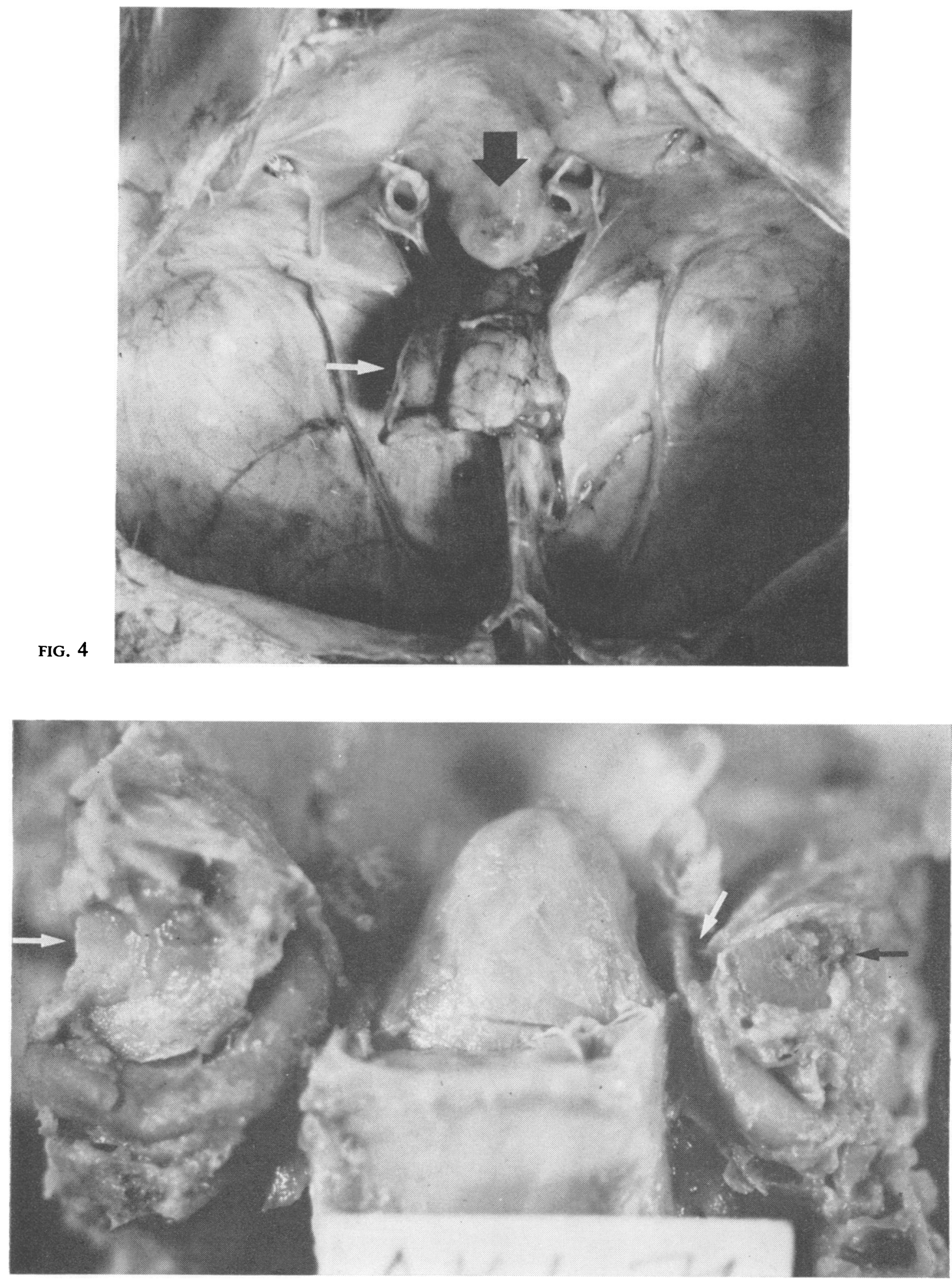

FIG. 5 


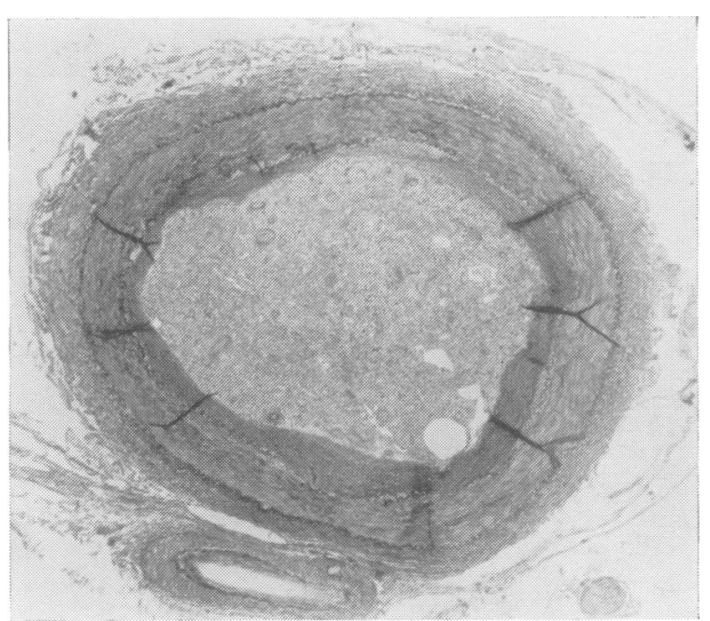

FIG. 6. Case 2. Left vertebral artery just above the main atheromatous lesion showing lumen occluded by a thrombus undergoing organization and early canalization. Verhoeff, $\times 1.5$.

remained in a state of 'coma vigil' until death two weeks later.

Necropsy showed recent infarction symmetrically in both posterior cerebral artery territories. In addition, there were scattered periaqueductal infarcts throughout the tegmentum of the brain-stem, infarction in the cerebellum bilaterally, and infarc- tion of the upper cervical cord (Figs 1 and 2). The odontoid process was displaced to the right and was herniated upwards for a distance of $1 \mathrm{~cm}$ through the foramen magnum (Figs 3, 4, and 5). As a result, the right vertebral artery was markedly narrowed and constricted in an hourglass fashion between the tip of the odontoid process and the lip of the foramen magnum. The left vertebral artery was recently thrombosed in its mid-cervical portion at a site of $90 \%$ atheromatous stenosis (Fig. 6).

Significant general findings in addition to rheumatoid arthritis were severe bilateral fibrotic lung disease with silicotic nodules (some showing central necrosis), emphysema, nephrosclerosis, right and left ventricular hypertrophy, and generalized lymphadenopathy.

SUMMARY The suboccipital head pain, muscular weakness, and paraesthesia seem related to the mechanical displacement of $\mathrm{C} 1$ and $\mathrm{C} 2$ vertebrae, causing pressure on the greater occipital nerve and compression of the cervical cord by the posteriorly displaced odontoid process. The clearly isolated symptoms of vertigo on head flexion or rotation suggest brain-stem ischaemia but could alternatively $\stackrel{\mathbb{D}}{\mathrm{D}}$ be explained by odontoid pressure on the medulla을 oblongata. The history of blackouts, poorly wit nessed, although likely to be secondary to orthostatic hypotension might also reflect intermittent vertebro? basilar artery insufficiency from extrinsic vascularô. compression related to AAS. His terminal event occurred while in traction on a horizontal Stryker
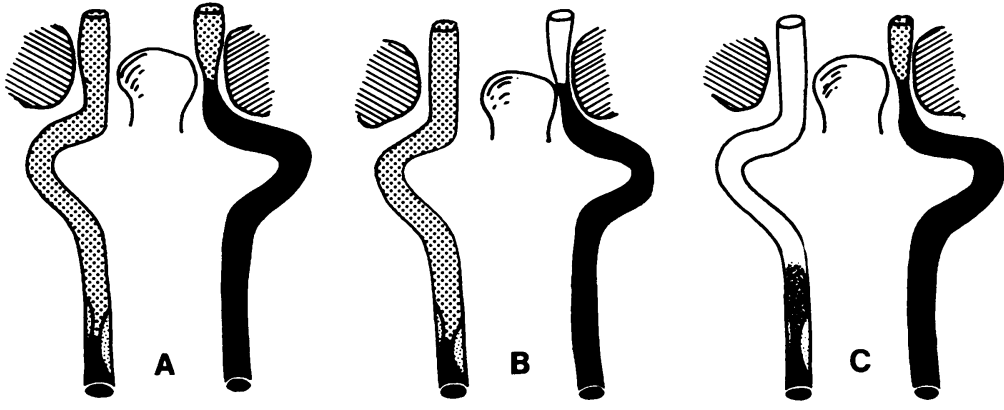

FIG. 7 Case 2. Diagram illustrating the reduced blood flow past the atheromatous stenosis on the left side and past the hourglass constriction between the eccentrically placed odontoid process and rim of the foramen magnum on the right side. A. With the long axis of the head in an anteroposterior position, without symptoms. B. Vertigo occurred on rotation of the head to the right side by further reducing vertebral blood flow. C. Thrombosis (lower left) of the left vertebral artery severely reducing flow to basilar artery. Infarction could have been precipitated by rotation as in $\mathrm{B}$ or could have occurred without rotation. 圆= reduced flow. $\square=$ no flow. $\square=$ normal flow. 
frame which prevented head flexion but allowed head rotation. Here the pathology would seem to be clearly vascular. The left vertebral artery in the previous 'stroke-like episode' 30 days earlier probably thrombosed, giving rise to insufficient basilar artery blood flow and therefore resulting in infarction. In the terminal stroke-like episode, the right vertebral artery was probably compressed during rotational movements further reducing the flow to the basilar artery and leading to an extension of the infarct (Fig. 7).

\section{DISCUSSION}

We feel these two patients with rheumatoid arthritis had paroxysmal symptoms of vertebrobasilar artery insufficiency related to change of head position. Both had gross degrees of instability and subluxation of their atlantoaxial joint and, in the second case, there was a significant degree of upward herniation of the odontoid process into the foramen magnum, pseudobasilar invagination (Martel, 1961).

Review of the literature would suggest that the finding of AAS is relatively frequent (Davis and Markley, 1951; Robinson, 1966; Stevens et al., 1971; Smith et al., 1972) but correlates poorly with neck symptomatology. Smith et al. (1972), in a large series collected over 10 years, found an incidence of $15 \%$ in 962 cases and Stevens et al. (1971) found an incidence of $25 \%$ in 100 successive rheumatoid patients attending an outpatient clinic. These authors and others have looked at the frequency of associated neurological involvement in patients with AAS. Smith et al. (1972) found $13 \%$ of those with AAS had evidence of spinal cord or brain-stem involvement, whereas Stevens et al. (1971) found 'myelopathy' to be present in $66 \%$ of their patients as judged by a pathological increase in deep tendon reflexes. They comment that limb strength and muscle tone could seldom be tested adequately because of pain or joint deformity.

Smith et al. (1972) in their paper entitled 'Natural history of rheumatoid cervical subluxations' found that 10 out of 130 patients with AAS developed neurological involvement over an average follow-up period of seven to eight years. Of this group, they felt that six developed features compatible with vertebral artery involvement but these patients were not studied by angiograms because the risks were not justified. Stevens et al. (1971) had one patient with paroxysmal brain ischaemia on neck flexion. Robinson (1966) reported on 22 patients with rheumatoid AAS and noted that three patients had signs of myelopathy. Four other patients had symptoms which may be interpreted as ischaemic. Two had what sounded like 'drop attacks' (Sheehan et al., 1960), one had vertigo, nausea, and vomiting associated with rotation of the head, and one had vertigo and blurred vision, also on head rotation.

Crellin et al. (1970) reported on 11 patients with severe rheumatoid AAS and neurological symptoms who were operated on. Two had symptoms of vertebral artery insufficiency, whereas the rest had symptoms of myelopathy.

The neurological complications of rheumatoid AAS usually take the form of a chronic compressive myelopathy from a backward slipping of the odontoid process onto the cervical cord. Clinically, these patients will have varying degrees of motor weakness, hyperreflexia, and Babinski responses. Sensory signs from this cord lesion are less frequent but patients who do have symptoms usually describe sensory paraesthesia on neck flexion. The other neurological syndrome which we feel these two cases exemplify is that of brain-stem ischaemia from vertebral artery compromise. Admittedly, both had true vertigo on head flexion or rotation which could be interpreted differently as possibly vestibular end-organ dysfunction; but both had the mechanism for impairing vertebral artery flow by increasing the tortuosity of the vertebral artery where it makes a double hairpin turn around the lateral mass of the $\mathrm{Cl}$ vertebra and, in effect, causing a dynamic stenosis by attenuating the vessel. Also the second case had severe atheromatous narrowing of his left vertebral artery and an hourglass constriction in the right vertebral artery at the level of the foramen magnum. His head on turning to the right would rotate about the odontoid process which would act as a fulcrum and therefore pinch off or reduce further the blood flow through this vessel in view of the abnormal anatomical arrangement (Fig. 7) from the upward herniation of the odontoid process into the foramen magnum.

It has been shown in a cadaver study (Toole and Tucker, 1960) that physiological changes in 
head position can lead to a reduction in vertebral artery and perhaps carotid blood flow. Similarly, in a human angiographic study (Sheehan et al., 1960) in patients with symptoms of vertebrobasilar artery insufficiency it was also possible to show a reduced or complete obstruction to blood flow through the vertebral artery with changes in head position. The majority of these patients had together a varying degree of atherosclerosis and cervical spondylosis with osteophytes compressing the vertebral artery usually suggesting additive factors accounting for these symptoms.

The gross dissection was the work of Dr M. J. Ball; Dr Colin Anderson prepared the spinal specimen.

\section{REFERENCES}

Chun, C. Kao, Messert, B., Winkler, S. S., and Turner, J. H. (1974). Rheumatoid C1-C2 dislocation: pathogenesis and treatment reconsidered. Journal of Neurology, Neurosurgery, and Psychiatry, 37, 1069-1073.

Crellin, R. Q., MacCabe, J. J., and Hamilton, E. B. D. (1970). Severe subluxation of the cervical spine in rheumatoid arthritis. Journal of Bone and Joint Surgery, 52, 244-251.
Davis, F. W., and Markley, H. E. (1951). Rheumatoid arthritis with death from medullary compression. Annals of Internal Medicine, 35, 451-454.

Martel, W. (1961). The occipito-atlanto-axial joints in rheumatoid arthritis and ankylosing spondylitis. The American Journal of Roentgenology, 86, 223-240.

Mathew, N. T., and Meyer, J. S. (1974). Pathogenesis and natural history of transient global amnesia. Stroke, 5 , 303-311.

Robinson, H. S. (1966). Rheumatoid arthritis-atlanto-axial subluxation and its clinical presentation. Canadian Medical Association Journal, 94, 470-477.

Sheehan, S., Bauer, R. B., and Meyer, J. S. (1960). Vertebral artery compression in cervical spondylosis. Neurology (Minneap.), 10, 968-985.

Smith, P. H., Benn, R. T., and Sharp, J. (1972). Natural history of rheumatoid cervical luxation. Annals of the Rheumatic Diseases, 31, 431-439.

Stevens, J. C., Cartilidge, N. E. F., Saunders, M., Appelby, A., Hall, M., and Shaw, D. A. (1971). Atlanto-axial subluxation and cervical myelopathy in rheumatoid arthritis. Quarterly Journal of Medicine, 40, 391-408.

Toole, J. B., and Tucker, S. H. (1960). Influence of head position upon cerebral circulation. Archives of Neurology, 2, 616-622.

Webb, F. W S., Hickman, J. A., and Brew, D. St. J. (1968). Death from vertebral artery thrombosis in rheumatoid arthritis. British Medical Journal, 2, 537-538. 